\title{
Efectividad del tratamiento de la enfermedad de Dupuytren (ED) mediante fasciotomía enzimática con colagenasa. Estudio multicéntrico cohortes prospectivo
}

\author{
A. García Olea ${ }^{\mathrm{I}}$, A. F. Dudley ${ }^{2}$, M. D. Gimeno Andrade 3 , M. Ocampos ${ }^{4}$, \\ H. Fahandezh-Saddi ${ }^{5}$, M. Del Cerro ${ }^{6}$ \\ ' Servicio de Traumatología. Hospital Puerta de Hierro Majadahonda, Madrid. ²Servicio de Traumatología. \\ Hospital Universitario La Princesa, Madrid. ${ }^{3}$ Servicio de Traumatología. Hospital Clínico San Carlos, Madrid. \\ ${ }^{4}$ Servicio de Traumatología. Hospital Infanta Leonor, Madrid. ${ }^{5}$ Servicio de Traumatología. \\ Fundación Hospital Alcorcón, Madrid. 'Unidad de Cirugía de Mano. Hospital Beata María Ana, Madrid.
}

\begin{abstract}
Resumen: Objetivos: Se ha realizado un estudio prospectivo multicéntrico con el objetivo de evaluar la efectividad del tratamiento de la enfermedad de Dupuytren (ED) con colagenasa mediante la medición de la corrección de la contractura en flexión de las articulaciones tratadas con colagenasa y la tasa de recidivas al mes, 6 y 12 meses.

Material y métodos: Se evaluaron los sujetos que presentasen contractura de $20^{\circ}$ o mayor en articulaciones metacarpofalángicas (MCF) o interfalángicas proximales (IFP). Se recogieron datos al mes, 6 y 12 meses postinfiltración. Se compararon las medias entre grados de contractura y su evolución en el tiempo.

Resultados: Los casos con afectación exclusiva MCF sumaron 58 , su contractura media fue de $50^{\circ}$. Los pacientes tratados de IFP fueron 16, su contractura media fue de $64^{\circ}$. Aquellos que tenían patología en ambas articulaciones fueron 52 casos. La MCF obtuvo una extensión completa al mes en el $80 \%$ y la IFP en el $66 \%$ de casos con contractura menor de $50^{\circ}$. La MCF y el $33 \%$ de las IFP menor de $40^{\circ}$ mantuvieron la corrección al año.

Conclusiones: Los autores recomiendan el tratamiento mediante colagenasa en pacientes que presentan contractura en flexión de un solo dedo y que afecta a la articulación MCF.
\end{abstract}

Palabras claves: Colagenasa Clostridium Histolyticum, Contractura Dupuytren, Injección.

\section{Collagenase injection effectiveness for Dupuytren contracture. Multicen- ter prospective cohort study}

\begin{abstract}
Objectives : We performed a prospective multicenter study to evaluate the effectiveness of the treatment of Dupuytren Disease with collagenase by measuring the correction of flexion contracture of the collagenase-treated joints and the relapse rate at Ist, 6 th and 12 th months.

Material and methods: Subjects with contracture of $20^{\circ}$ or higher in metacarpophalangeal (MCP) or proximal interphalangeal (PIP) were evaluated. Data at month, 6 and 12 months were collected. Means between degrees of contracture and its evolution over time were compared.

Results: Cases involving only MCP amounted to 58 , its average contracture was $50^{\circ}$. PIP Patients were 16 and its average contracture was $64^{\circ}$. After reviewing the post-injection data, MCP obtained a full extension in $80 \%$ and the PIP in $66 \%$ of cases with less contracture of $50^{\circ}$. MCP and $33 \%$ of PIP with less of $50^{\circ}$ remained under correction after the Ist year.

Conclusions: The authors recommend treatment with collagenase in patients with flexion contracture of only a finger with a pretendinous clearly palpable cord that affects exclusively to the MCP joint .
\end{abstract}

Key words: Collagenase Clostridium histolyticum; Dupuytren contracture, injection.

La Enfermedad de Dupuytren (ED) se caracteriza por ser un trastorno fibroproliferativo progresivo que transforma el esqueleto fibroso de la mano en un tejido patológico, que llamamos nódulos y cuerdas y provocan una contractura en flexión de los dedos.

La ED a menudo limita la función y actividades de la vida diaria (20\% de los pacientes que consultan) ', incluyendo la higiene personal, la posibilidad de introducir la mano en un bolsillo o guante, estrechar la mano de otra persona y lleva a muchos pacientes a buscar tratamiento. La ED se trataba casi exclusivamente de forma quirúrgica. La tasa de recurrencias y morbilidad asociados al tratamiento quirúrgico de la

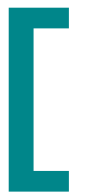

Avenida paralela, 43. 28221. Majadahonda. Madrid garcialerma@msn.com 
ED es muy variable según los trabajos publicados, pero considerablemente alta ${ }^{1-7}$. Tanto las recidivas como las complicaciones asociadas al tratamiento quirúrgico provocan una alta incidencia de reintervenciones y elevados gastos hospitalarios. Existen numerosos trabajos que han relacionado la gravedad de la contractura con la severidad de las complicaciones y también está demostrado que el porcentaje de complicaciones mayores como lesiones de pedículos vasculonerviosos, necrosis cutáneas o infecciones y distrofia se duplican en los casos de cirugías de la recidiva ${ }^{4-7}$. Además, algunos pacientes no pueden someterse a cirugía por su edad avanzada o enfermedad coexistente. Todos estos motivos han sido el motor para la búsqueda de un tratamiento no quirúrgico que sea eficaz, con escasa morbilidad y cuyos costes no sean mayores al tratamiento previo ${ }^{8}$.

Otros tratamientos no quirúrgicos como radioterapia, inyección de corticoides, vitaminas, etc. habían supuesto un fracaso hasta la aparición de la fasciotomía enzimática; que fue publicada por Hueston y cols. en 1971, utilizando enzimas no específicas como la tripsina y la hialuronidasa ${ }^{2,3}$. Dado que las cuerdas se componen principalmente de colágenos tipo I y III, la investigación se ha centrado en el desarrollo de la enzima colagenasa ${ }^{2,3,9-14}$. La colagenasa procedente del Clostridium histolyticum es, a fecha de hoy, el único tratamiento farmacológico que ha sido aprobado por la European Medicine Agency como terapia de la ED. En España, se está utilizando la colagenasa desde junio de 2011 .

El presente trabajo se diseñó como estudio multicéntrico (de cohortes clásico) longitudinal prospectivo con el objetivo de evaluar la efectividad del tratamiento de la ED con colagenasa mediante la medición de dos parámetros principales:

a.- La corrección de la contractura en flexión de las articulaciones metacarpofalángica (MCF) y/o interfalángica proximal (IFP) tratadas con colagenasa.

b.- La tasa de recidivas al mes, 6 y 12 meses.

Se realizó además un estudio demográfico de nuestra población.

\section{MATERIALY MÉTODO:}

\section{Criterios de inclusión}

I.- Mayores de 18 años de edad.
2.- Presentar una contractura de Dupuytren de al menos $20^{\circ}$ (signo del escritorio positivo) causada por una cuerda palpable en al menos un dedo que no sea el pulgar.

3.- Los pacientes mujeres que potencialmente puedan quedar embarazadas deben utilizar un método de contracepción de alta efectividad. Un test de embarazo estaría indicado si fuera preciso.

4.- A todos los pacientes se les explicó la patología y por qué eran subsidiarios de tratamiento. Se ofrecieron los dos posibles tratamientos a realizar; a saber, quirúrgico o enzimático. Se explicaron los pros y contras de cada uno. Los pacientes que elegían el tratamiento enzimático firmaron el correspondiente consentimiento informado autorizado por el Comité Ético de los respectivos centros. Todos los pacientes fueron informados que iban a ser objeto de un estudio clínico prospectivo de acuerdo a los principios de la Declaración de Helsinki.

\section{Criterios de exclusión}

I.- Pacientes en tratamiento con anticoagulantes 0 que hayan recibido medicación anticoagulante, aquellos que no habían realizado correctamente el cambio a heparina de bajo peso molecular 7 días antes de la terapia y los que hubiesen recibido doxiciclina 15 días antes.

2.- Pacientes embarazadas, lactantes o que tengan previsto quedarse embarazadas durante el tratamiento.

3.- Pacientes con hipersensiblidad sistémica a la colagenasa o cualquiera de los excipientes del producto.

4.- Pacientes con enfermedades musculares crónicas, neurológicas o neuromusculares que afecten las manos.

5.- Se excluyeron del tratamiento con colagenasa todos aquellos pacientes en postoperatorio de la ED u otra patología de la mano.

6.- También fueron descartados aquellos pacientes cuyas manos tuviesen más de 2 dedos con flexión de las articulaciones MCF o IFP y las articulaciones con una flexión mayor de $95^{\circ}$, además de aquellos casos de recidiva de la flexión articular sin una cuerda claramente palpable. 


\section{Diseño clínico / intervención}

Todos los pacientes fueron tratados en Unidades de Cirugía Ambulatoria o en el ámbito de la propia consulta externa según la organización interna de cada uno de los centros. Se administró la sustancia en las dosis y puntos de inyección que recomienda la técnica del prospecto del medicamento (Xiapex®). En todos los centros las infiltraciones fueron realizadas por cirujanos con amplia experiencia en la patología de la mano y habían aprobado un curso de capacitación certificado por la empresa distribuidora. De forma habitual, siempre que coexistía afectación de articulaciones MCF e IFP en un mismo dedo se realizaba una primera infiltración en la MCF a semejanza del tratamiento habitual de la ED mediante técnica quirúrgica. De forma excepcional ha sido necesario infiltrar la IFP en un segundo tiempo debido a la alta satisfacción de paciente y cirujano con los resultados tras la primera infiltración y a la dificultad posterior para identificar una cuerda claramente palpable.

En el caso de los 27 pacientes tratados en uno de los centros, se monitorizó la infiltración en la cuerda mediante el uso de ecografía.

Se realizó un vendaje preventivo del uso de la mano hasta la extensión del dedo y rotura de la cuerda. La segunda parte del tratamiento se llevó a cabo previa infiltración con anestesia locorregional en la cara palmar de la muñeca unos 30 minutos antes de la extensión forzada del dedo.

Se estableció un régimen de visitas postratamiento a los 10 días, 30 días y a los 6 y 12 meses.

Se definió el éxito terapéutico como la contractura en flexión (o déficit de extensión) de la articulación menor o igual a $5^{\circ}$ al mes del tratamiento y la recidiva como la reaparición de un déficit de extensión superior a $20^{\circ}$ o pérdida de al menos $20^{\circ}$ en la extensión obtenida. En todos los casos, para realizar la medición se utilizó un goniómetro de dimensiones ajustadas para poder apoyar adecuadamente los brazos de la herramienta al dorso de las falanges o metacarpianos y se solicitó al paciente que ejecutase una extensión activa de sus dedos.

Se realizó una tabla común para todos los centros que recogía una serie de variables de conocida relación con una mayor propensión a desarrollar la ED, mano, dedo y articulación afectos, éxito del tratamiento realizado y efectos secundarios. Respecto del tratamiento se recogieron datos al mes, 6 y 12 meses postinfiltración y posteriormente se recogerán datos anuales durante un periodo de 5 años. Se definieron y registraron los diversos grados de edema + /- dolor +/-hematoma y/o flictenas en el punto de inyección según terminología común de contusión. Se cuantificaron aquellos casos en los que aconteció un rasgado de la piel tras realizar la extensión del dedo. No se registraron otros efectos adversos debido a su escasa incidencia.

Se acotó la inclusión de pacientes en el estudio a un periodo de tiempo de 18 meses, entre 1 de junio de 2011 y 30 de noviembre de 2012. Los datos eran incorporados a la tabla común vía e-mail. () Tabla I.

\section{Características demográficas de la población}

La edad media de los pacientes fue de 64 años, con un rango de edad que fue de los 33 a los 89 años. Los pacientes mayores de 75 años fueron el $21 \%$ de la muestra y los menores de 45 (factor considerado predisponente de peores resultados) sumaron el II\%. Las mujeres tratadas fueron II, lo que supone el $7 \%$ de la muestra. Un $16 \%$ de la población estudiada había sido previamente tratados mediante cirugía.

Si estudiamos los factores considerados diatésicos o favorecedores de peores resultados y mayor gravedad de la ED, observamos que en la población estudiada el 83\% tiene 2 o menos de dichos factores. A destacar que los pacientes menores de 45 años presentaban 3 o más factores. (a) Tabla II.

\section{Análisis estadístico}

Para el análisis estadístico, se calcularon parámetros descriptivos estándar: media y desviación estándar (DE) para las variables continuas y $n^{\circ}$ de casos y porcentajes para las variables categóricas. La distribución normal se probó mediante las pruebas de Kolmogorov-Smirnov. Los grados de contractura de las articulaciones y su evolución en el tiempo después de I, 6 y 12 meses se compararon mediante la prueba t de Student para muestras apareadas, expresando el resultado con el cambio medio y el intervalo de confianza del $95 \%$. Para todas las pruebas se aceptó un valor de significación inferior a 0,05 en contraste bilateral. Igualmente, se comparó la población del hospital en el cual se realizaron las infiltraciones guiadas por ecografía con el resto de hospitales. Los resultados tras el tratamiento con colagenasa también se compararon mediante la realización de un test de $t$ de Student. Se utilizó el programa estadístico SPSS v. 14.0 (SPSS Inc. IL , EE.UU. ). 


\section{TABLA I - TABLA DE RECOGIDA BÁSICA DE DATOS.}

\section{DATOS FILIACIÓN PACIENTE}

HOSPITAL

NOMBRE PACIENTE

$\mathrm{NHC}$

LADO

EDAD

PROFESIÓN

ACTIVIDAD MANUAL

LIGERA MODERADA

ALTA

\section{FACTORES DIATESIS}

FUMA

ALCOHOL

NO SI

EPOC

NO BAJO

BAJO

MODERADO

ALTO

DIABETES MELLITUS

HEPATOPATÍA

$<45$ años

PEYRONIE

LEDDERHOSE

NÓDULOS GARROD

BILATERAL

ANTECEDENTES FAMILIARES

SUMA FACTORES DIATESIS

ANAMNESIS ENFERMEDAD

DURACIÓN ENFERMEDAD

$<1$ AÑO

$<3$ AÑOS

$<5$ AÑOS

$<7$ AÑOS

$<10$ AÑOS

$>$ 10 AÑOS

CIRUGÍA PREVIA DEDO

EXPLORACIÓN ENFERMEDAD

\begin{tabular}{|c|c|c|c|c|c|c|}
\hline DEDO AFECTO & $\|$ & III & IV & v & PULGAR & $1^{\mathrm{a} C O M I S U R A}$ \\
\hline ARTICULACIÓN AFECTA & MCF & $\mathbb{I F P}$ & & & & \\
\hline GRADOS CONTRACTURA & & & & & & \\
\hline ARTICULACIÓN VECINA? & & & & & & \\
\hline
\end{tabular}

1 MES

DEDO AFECTO

ARTICULACION AFECTA

GRADOS CONTRACTURA

ARTICULACION VECINA?

6 MESES

DEDO AFECTO

ARTICULACIÓN AFECTA

GRADOS CONTRACTURA

ARTICULACIÓN VECINA?

\section{MESES}

DEDO AFECTO

ARTICULACIÓN AFECTA

GRADOS CONTRACTURA

ARTICULACIÓN VECINA?

EFECTOS SECUNDARIOS

CONTUSIÓN (edema y/o hematoma y/o dolor)

CIRCUNSCRITO

DORSO MANO

MANO

EXTREMIDAD

LINFADENOPATÍA

RASGADO CUTANEO

OTROS 


\begin{tabular}{|c|c|c|c|}
\hline \multicolumn{4}{|c|}{ TABLA II - RESUMEN DATOS DEMOGRÁFICOS SIGNIFICATIVOS MUESTRA } \\
\hline & $\mathbf{N}$ & $\%$ & $\mathbf{N}^{0}$ casos total \\
\hline PACIENTES TRATADOS COLAGENASA & 151 & & \\
\hline DEDOS & 161 & & \\
\hline ANULAR & 78 & 48,40 & 161 \\
\hline MEÑIQUE & 73 & 45,30 & 161 \\
\hline MEDIO & 3 & 1,90 & 161 \\
\hline ÍNDICE & 2 & 1,20 & 161 \\
\hline PULGAR / I ${ }^{\circ}$ COMISURA & 5 & 3,30 & 161 \\
\hline EDAD MEDIA: 64 (33-89) & & & \\
\hline$>75$ años & 32 & 21,20 & 151 \\
\hline$<45$ años & 17 & 11,20 & $15 \mid$ \\
\hline MUJERES & 11 & 7,30 & 151 \\
\hline \multicolumn{4}{|l|}{ DESCARTADOS: } \\
\hline CIRUGÍA PREVIA & 24 & 15,90 & $15 \mid$ \\
\hline MCF + IFP, INFILTRADAS AMBAS & 4 & 2,60 & $|5|$ \\
\hline DATOS TABLA INCOMPLETOS & 4 & 2,60 & $15 \mid$ \\
\hline FRACASO TÉCNICA & 3 & 1,90 & $|5|$ \\
\hline \multicolumn{4}{|l|}{ TIEMPO EVOLUCIÓN } \\
\hline Menor 5 años & 9 & 7,10 & 126 \\
\hline Hasta 5 años & 68 & 53,90 & 126 \\
\hline Entre 5 y 7 años & 30 & 23,80 & 126 \\
\hline 10 años o más & 19 & 15,10 & 126 \\
\hline \multicolumn{4}{|l|}{ FACTORES DIATÉSICOS } \\
\hline INGESTAALCOHOL & 88 & 70,10 & 126 \\
\hline DIABETES & 36 & 28,60 & 126 \\
\hline FUMADOR & 19 & 15,00 & 126 \\
\hline AFECTACIÓN BILATERAL & 61 & 48,40 & 126 \\
\hline ANTECEDENTE FAMILIAR & 44 & 34,90 & 126 \\
\hline NÓDULOS DE GARROD & 6 & 4,80 & 126 \\
\hline PEYRONNIE & 0 & - & 126 \\
\hline LEDDERHOSE & 4 & 3,20 & 126 \\
\hline SUMA 20 menos & 105 & 83,30 & 126 \\
\hline SUMA 30 más & 21 & 16,60 & 126 \\
\hline
\end{tabular}

\section{RESULTADOS}

Entre junio de 2011 y noviembre de 2012 se han tratado en los diferentes centros que participaron en el estudio un total de $|6|$ dedos en $15 \mid$ pacientes.

El anular y meñique, con 78 y 73 casos respectivamente, fueron los dedos con mayor incidencia. El resto de dedos han sido tratados en número testimonial de casos; 5 en pulgar / primera comisura, 3 infiltraciones en dedo medio y 2 para el índice. () Figura I.

Si descontamos por criterios de exclusión los casos de tratamiento en pulgar / primera, comisura restan 156 dedos en 148 pacientes, de los cuales se han desesti-
NNo de infiltraciones

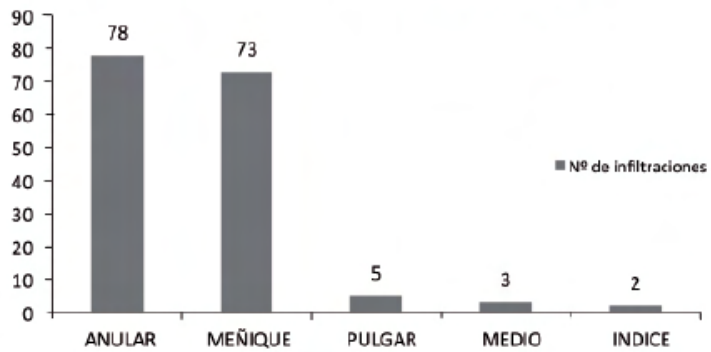

Figura $1 . N^{\circ}$ de total infiltraciones efectuadas.

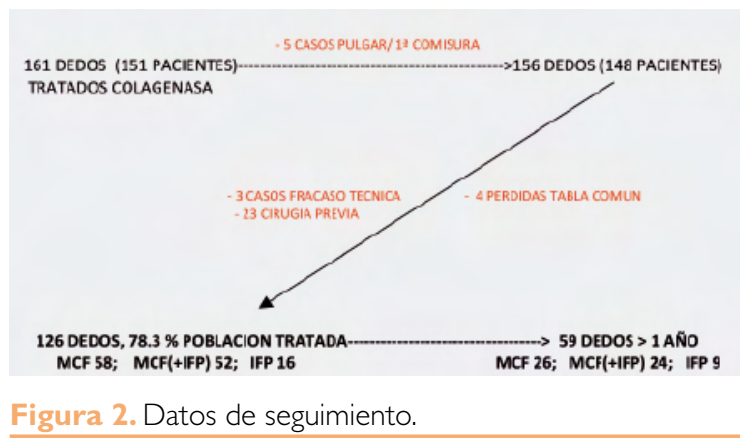

mado para estudios estadísticos los casos en los que no se rellenaron todas las casillas de datos demográficos o de las revisiones postratamiento, los dedos infiltrados en ambas articulaciones, los casos de cirugía previa y 3 casos en los que no se pudo conseguir la extensión de la articulación afecta al día siguiente de aplicar la colagenasa (uno de ellos por dolor en el punto de inyección y en los otros dos casos por mala técnica de preparación del liquido de infiltración). Así pues, se han realizado los correspondientes estudios estadísticos sobre un total de 126 dedos, el $78.3 \%$ de los casos tratados inicialmente. Del total de casos, al momento de remitir el presente trabajo se habían completado en la tabla común los datos del seguimiento año de tratamiento en 59 casos (46.8\%). (1) Figura 2.

Si estudiamos los datos preinyección respecto de las articulaciones afectas; los casos con afectación exclusiva de la MCF sumaron un total de 58, el valor de la media de su déficit de extensión fue de $50^{\circ}$ (rango $20-90^{\circ}$ ). En el $60 \%$ de los casos la contractura en flexión fue menor de $40^{\circ}$. Los pacientes tratados únicamente de la articulación IFP fueron 16, su valor medio para el déficit de extensión fue de $64^{\circ}$ (rango $20-90^{\circ}$ ). En un 30\% de los casos con afectación de IFP la contractura en flexión fue igual o menor de $40^{\circ}$. Aquellos que tenían patología en 


\section{Tabla III - Grados de contractura articular (Media +/- DE)}

\begin{tabular}{|c|ccccccccccc}
\hline & BASAL (N= ) & I MES (N= ) & \% mejoría BASAL & 6 MESES (N= ) & \% mejoría BASAL & I2 MeSES (N= ) & \% mejoría BASAL \\
\hline MCF & $40.9+/-16.258$ & $1.6+/-7.0$ & 58 & $95,60 \%$ & $2.8+/-8.2$ & 58 & $92,60 \%$ & $5.4+/-11.3$ & 26 & $95,80 \%$ \\
\hline MCF + IFP & $49.9+/-24.6$ & 52 & $2.6+/-9.6$ & 52 & $95,20 \%$ & $3.9+/-11.1$ & 52 & $91,40 \%$ & $6.3+/-13.7$ & 24 & $87,40 \%$ \\
\hline IFP & $64.2+/-21.0$ & 16 & $14.4+/-14.2$ & 16 & $77,40 \%$ & $26.7+/-14.8$ & 16 & $63,80 \%$ & $41.7+/-25.6$ & 9 & $56,30 \%$ \\
\hline
\end{tabular}

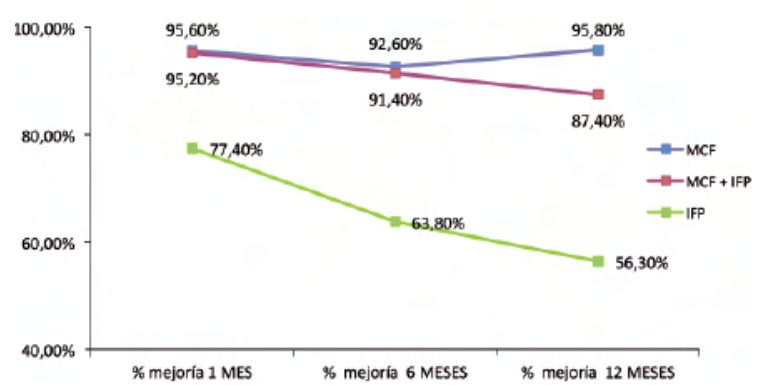

Figura 3. Porcentaje de mejoría contractura articular respecto basal.

ambas articulaciones fueron un total de 52 casos, sus medias para el déficit de extensión fueron de $40^{\circ}$ y $50^{\circ}$ respectivamente.

Al estudiar los mismos datos de afectación articular postinyección; se objetiva que en la MCF se obtiene una ausencia completa de déficit de extensión y que esta corrección se mantiene en el tiempo o tiene una mínima pérdida; con un valor medio de déficit de extensión al año de tratamiento de 5,4. Por el contrario, la IFP obtuvo peores resultados tanto inmediatos como después de varios meses. Así, la media de grados de déficit de extensión al mes, 6 meses y al año fue incrementando desde los $14.4^{\circ}$ a $26.7^{\circ}$ y $41.7^{\circ}$ respectivamente. Este comportamiento se objetiva también en aquellos casos con afectación de ambas articulaciones; el deterioro de la corrección es más rápido para el caso de la articulación IFP. Si reflejamos en gráficos el deterioro del porcentaje de mejoría respecto del tiempo, se nos muestra claramente este comportamiento para cada una de las localizaciones de lesión (a) Figura 3. () Tabla III.

Se realizó un test de t de Student para datos pareados respecto de los grados de contractura de las articulaciones MCF e IFP a los 6 y 12 meses postinfiltración, los resultados obtenidos muestran la menor duración del efecto de la colagenasa en el caso de la articulación IFP. Así, para los pacientes que tiene afecta la articulación MCF si se comparan de forma pareada

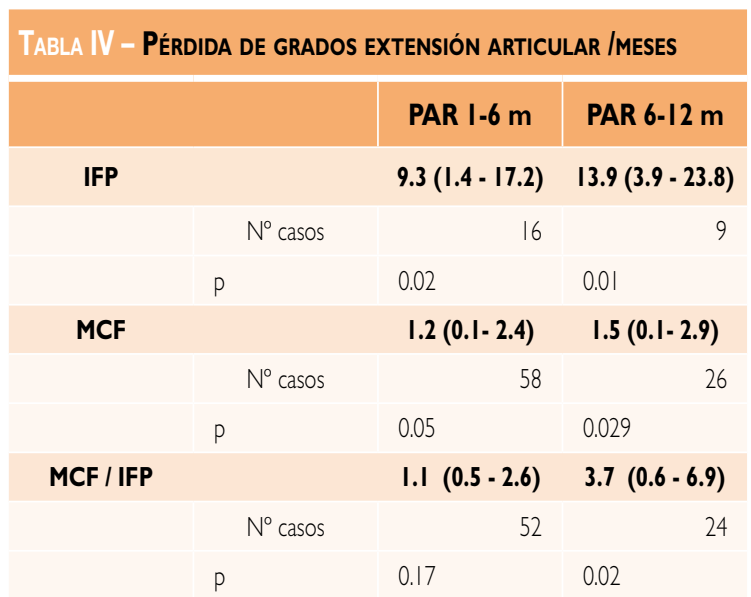

Datos expresados en Media (Intervalo confianza 95\%).

los datos al mes y 6 meses postinfiltración y entre los 6 y 12 meses, el deterioro de la corrección articular es menor de 1,5 ${ }^{\circ}$ de valor medio. () Tabla IV. En el caso concreto de pacientes que tienen afecta la articulación IFP, si hacemos la misma comparación de datos pareados, se obtiene un claro aumento del déficit de extensión tanto si sólo está afecta la articulación IFP (9,3 y I 3,9 respectivamente) como si están enfermas MCF e IFP.

Igualmente, se compararon las poblaciones del hospital en el cual se realizaron las infiltraciones guiadas por ecografía y las del resto de hospitales sin encontrar diferencias en las muestras salvo para el valor medio de los grados de contractura MCF $(p<0.01)$. Los resultados tras el tratamiento con colagenasa también se compararon mediante la realización de un test de $\mathrm{t}$ de Student, hallando que no hay diferencia alguna por el hecho de usar el ecógrafo. () Tabla $V$ y (8) Tabla VI.

Respecto de los efectos secundarios, todos los pacientes tuvieron contusión local (edema-hematoma y dolor) en diferentes gradaciones que no precisaron de tratamiento adicional. Los pacientes referían resolución de dichos efectos adversos en la consulta de los 


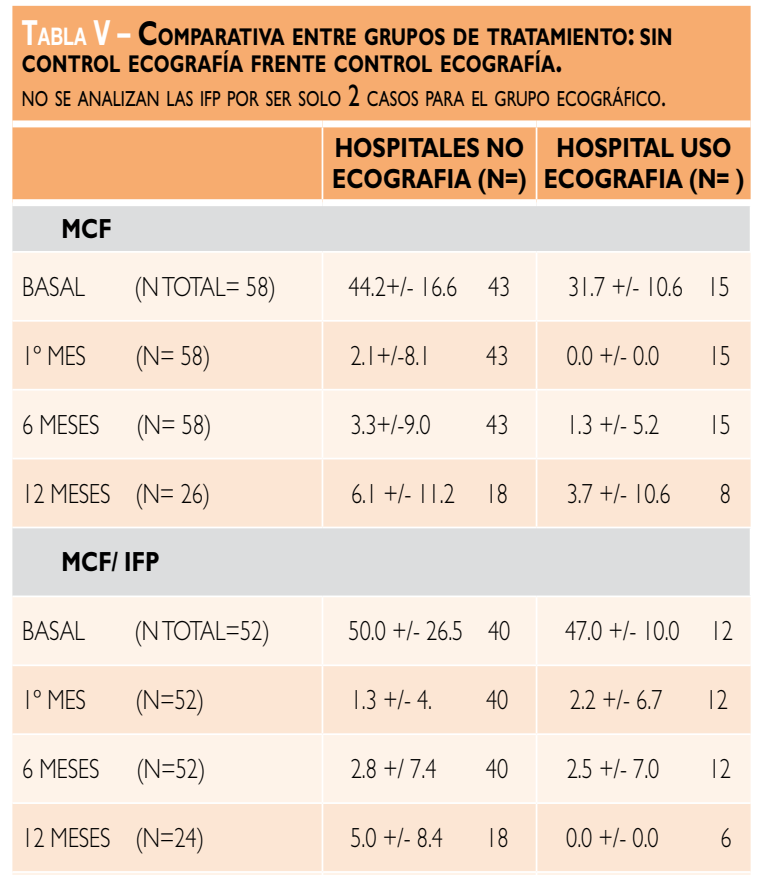

Grados de contractura articular

(Media +/- DE)

Grados de contractura articular (Media +/- DE)

I0 días. En ningún caso la reanudación de la terapia anticoagulante supuso empeoramiento del hematoma local en el punto de inyección.(Figura 4. La laceración cutánea ocurrió en 20 casos (15,9\%), no fue preciso realizar tratamiento quirúrgico y en todos los casos, la mano presentaba una cicatrización completa en un periodo máximo de 3 semanas. () Figura 5.

En un caso no se consiguió la extensión por dolor muy intenso en el punto de inyección y no permitir el paciente que se realizase la extensión del dedo; paciente que luego hubo de ser tratado quirúrgicamente por disestesias y parestesias. En otros

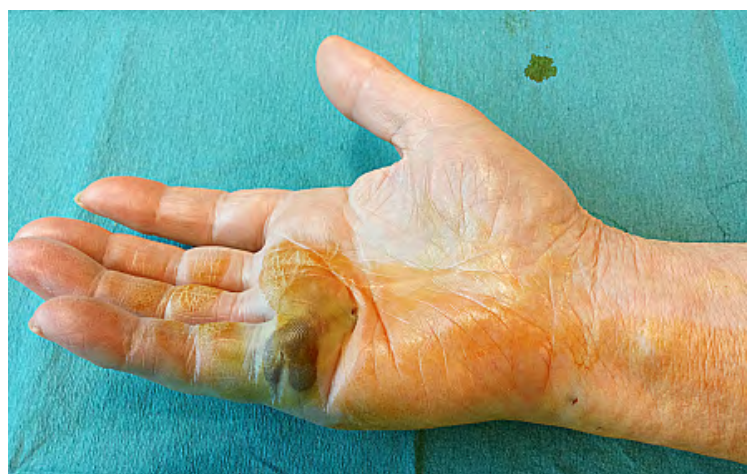

Figura 4. Efectos adversos típicos postinfiltración de colagenasa: edema, hematoma y dolor en punto de inyección.

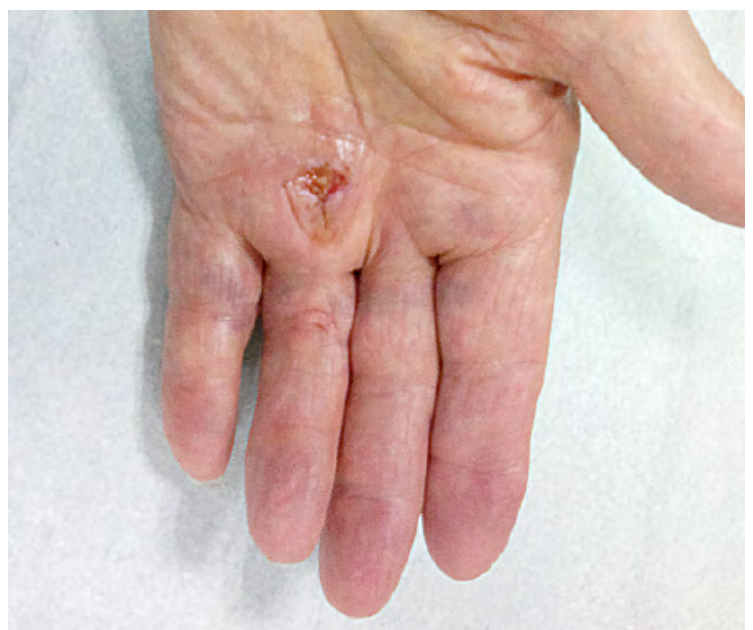

Figura 5. Laceración cutánea a los 4 días tras la maniobra de extensión.

2 pacientes afectos de contractura en MCF y en los que se consiguió el éxito terapéutico, se ha tenido que realizar un tratamiento quirúrgico a los pocos meses por disestesias y parestesias distales al punto de inyección. En todos los casos, el cirujano no encontró datos macroscópicos de lesión en el

\begin{tabular}{|c|c|c|c|c|c|}
\hline & BASAL & I MES & & MESES & I 2 MESES \\
\hline MCF & $12.5(3.3-21.8)$ & $2.1(0.2-6.4)$ & 2.0 & $(0.3-6.9)$ & $2.4(0.8-12.4)$ \\
\hline $\mathrm{N}^{\circ}$ casos & 58 & 58 & & 58 & 26 \\
\hline $\mathrm{p}$ & 0.01 & 0.3 & 0.4 & & 0.6 \\
\hline MCF / IFP & $3.0(0.1-20.2)$ & $0.9(0.4-2.8)$ & 0.2 & $(0.5-6.0)$ & $5.0(0.3-13.9)$ \\
\hline $\mathrm{N}^{\circ}$ casos & 52 & 52 & & 52 & 24 \\
\hline$p$ & 0.7 & 0.6 & 0.9 & & 0.2 \\
\hline
\end{tabular}

Datos expresados en Media (Intervalo confianza 95\%). 

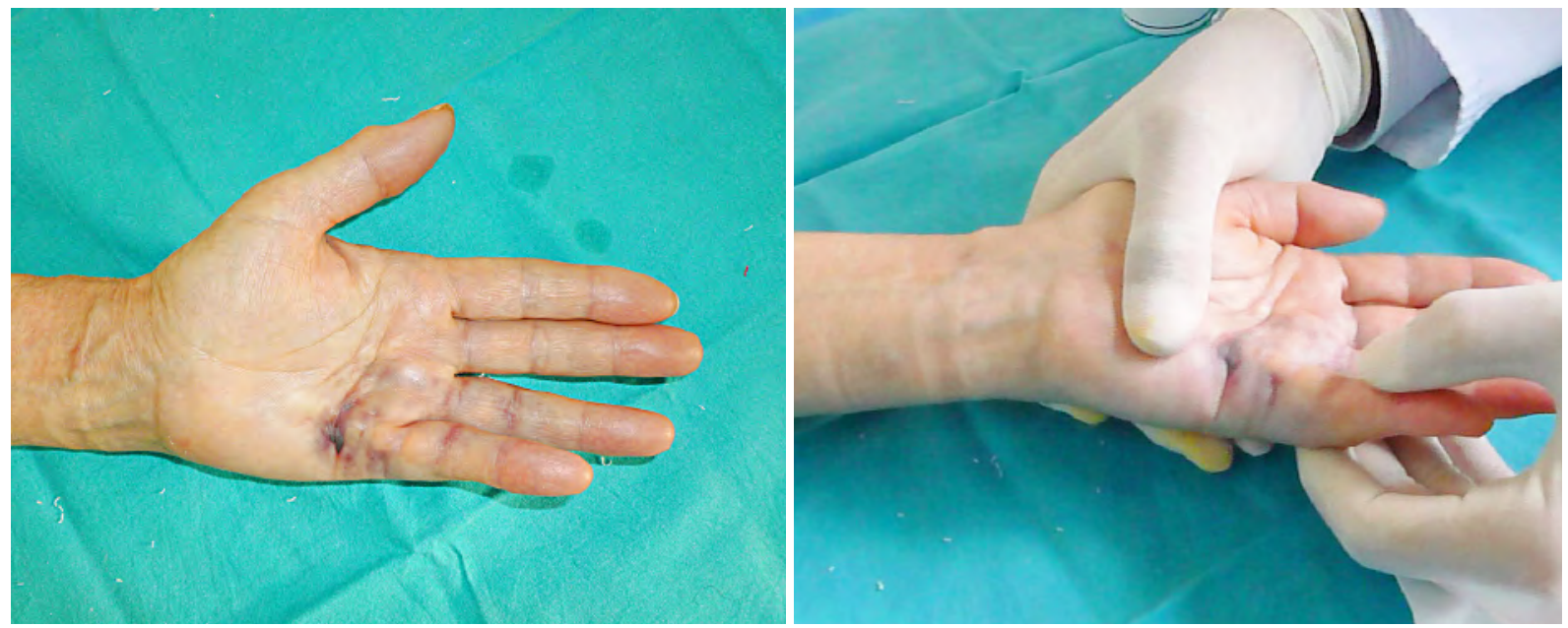

Figuras 6A y 6 B. Aspecto tras retirar vendaje a las 24 horas de infiltración MCF y previo a maniobra de extensión. B. Maniobra de extensión forzada MCF, se consigue extensión completa.

nervio y el cuadro se resolvió con la cirugía. Otros efectos secundarios que se refieren en ficha técnica acontecieron de forma excepcional y tampoco han necesitado de tratamiento.

\section{DISCUSIÓN}

Los autores del presente trabajo son conscientes de las limitaciones en las conclusiones debido al corto tiempo de seguimiento que afecta a una parte de la muestra, ya que sólo el 47 \% de los casos habían completado un periodo de estudio de I año. Es por ello que este estudio de debe considerar como preliminar.

Cabe destacar que en los estudios hasta ahora publicados por los autores de la técnica se realizó mayor número de infiltraciones por articulación y en la mayoría de casos, sólo separadas por 30 días ya que se buscaba la extensión completa de la articulación. En el estudio CORD I, los cirujanos establecieron el concepto de la articulación primaria y por ello las MCF duplicaron el número de casos respecto de las IFP'0. En los estudios CORD II $^{\prime \prime}$ y JOINT ${ }^{14}$, se evalúan los resultados tras un total de 12 y 9 meses respectivamente, pero igualmente las articulaciones han podido ser infiltradas varias veces y el porcentaje de éxitos no se relaciona respecto del tiempo desde la última infiltración. Así mismo, los autores de la técnica reconocen la dificultad de localizar una cuerda claramente palpable en IFP tras una segunda o incluso una primera infiltración (la media de infiltraciones por articulación no llegó a 2 en ninguno de los mencionados estudios) $)^{10-14}$. En nuestro estudio, con la intención de evaluar sólo articulaciones primarias y por facilidad de estudio estadístico, se han descartado los casos de infiltración secuencial o en dedos ya operados y se ha relacionado el tiempo de evolución respecto de la tasa de recidivas.

Así, cuando el paciente sólo tiene afectada la articulación MCF, en el 80 \% de casos se consigue la extensión completa y ésta se mantiene durante al menos el primer año. Figura 6. En el otro extremo está la articulación IFP; sobre la que hay que destacar que cuando el déficit de extensión es menor de $40^{\circ}$ al primer mes registra una extensión completa en el $66 \%$ de los casos pero presenta un deterioro de los resultados de extensión conseguidos ya en los primeros 6 meses. Después de I año, aquellos pacientes afectos de la IFP con menos de $40^{\circ}$ mantienen una contractura igual o menor de $20^{\circ}$ sólo en el $33 \%$ de los casos. Los resultados obtenidos en nuestro estudio son superponibles a otras series publicadas ${ }^{10-14}$. El estudio CORD I describe un porcentaje de éxitos del $77 \%$ para la articulación MCF y del $40 \%$ para IFP y el CORD II del 65 y $28 \%$ respectivamente. El estudio CORDLESS también refleja este comportamiento de MCF e IFP con un porcentaje de recidivas a los 3 años para la IFP del $56 \%{ }^{13,14}$ y el estudio JOINT II realizado en Europa y Australia refiere un porcentaje de éxito para MCF del 60 y del 32\% para IFP'4.

Estos resultados obtenidos para la IFP en nuestra serie y otras similares tienen explicación en la anatomía patológica macroscópica de la enfermedad. Si tenemos en cuenta que el tratamiento se realiza por 

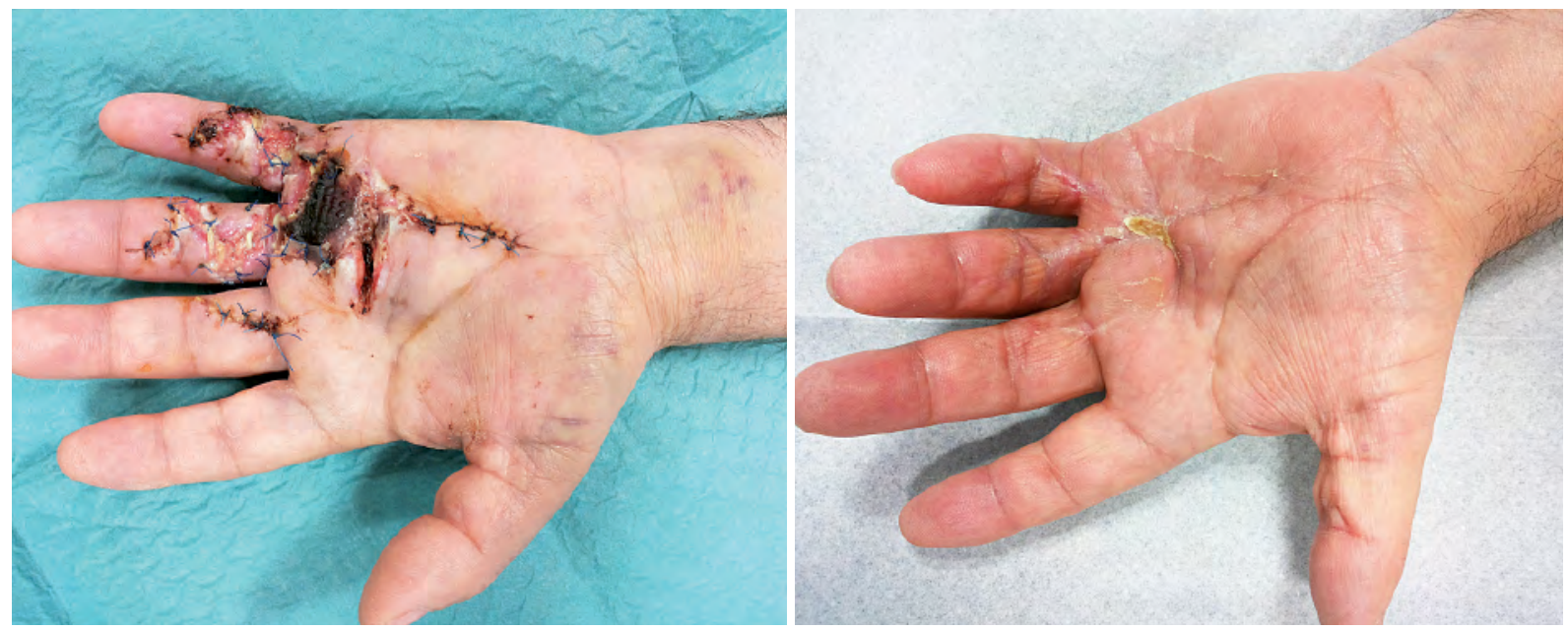

Figuras 7 A y 7 B. A. Efectos adversos de la cirugía en 3 radios con severas contracturas en flexión. A los 10 días se ha delimitado placa de necrosis en la palma. B. la misma mano I mes después de curas 2 veces por semana. Obsérvense las cicatrices y leve pérdida de extensión.

medio de una inyección en un espacio muy restringido, "de seguridad", siempre nos dejaremos zonas sin tratar. También las publicaciones de revisión de resultados quirúrgicos recogen este comportamiento en la IFP; en general y tanto para el tratamiento quirúrgico como para la colagenasa, a mayor contractura peores resultados tanto inmediatos como a medio plazo, mayor porcentaje de recidivas y en un plazo más corto $4-7,10-14$. Si a ello sumamos que los pacientes que tienen ya una afectación en el dedo-IFP son más jóvenes, tiene mayor número de factores diatésicos y mayor tiempo de evolución de la enfermedad (añadir factor articular por el tiempo en actitud flexo) y la afectación ocurre en el dedo meñique, se comprende que los resultados son peores y no se mantienen en el tiempo.

En uno de los hospitales de nuestra serie, se realizaba el tratamiento de infiltración asistido por ecografía, lo que no mejora los resultados pero ofrece mayor seguridad de no lesionar los pedículos vasculonerviosos. Los autores quieren destacar la escasa intensidad de los eventos adversos provocados por el tratamiento de la colagenasa, que están siempre presentes pero son bien tolerados por el paciente, mejoran de forma rápida sin interferir en la función de la mano y sólo si ocurre laceración cutánea precisan de revisiones de curas frecuentes durante unas 2 semanas. En el otro extremo encontramos el tratamiento quirúrgico, cuyos eventos adversos son menos frecuentes pero mucho más graves y en un gran número de casos dejan secuelas. (1) Figura 7 .

\section{CONCLUSIONES}

Los autores recomiendan el tratamiento de la ED mediante colagenasa en aquellos pacientes que presentan una contractura en flexión en un solo dedo con cuerda pretendinosa claramente palpable y que afecta de forma exclusiva a la articulación MCF. También aquellos pocos con afectación MCF de dedos vecinos por existir una cuerda pretendinosa palmar en $\checkmark$ oY. Si el paciente padece además alguna enfermedad grave que desaconseje el tratamiento quirúrgico o es mayor de 75 años y en aquellos en tratamiento anticoagulante, la colagenasa es el tratamiento de elección.

Por el contrario, se desaconseja como tratamiento en aquellos pacientes con afectación de más de 2 dedos y en aquellos con flexión mayor de $40^{\circ}$ de la IFP y con escasa o nula contractura de la MCF. Pacientes jóvenes, largo tiempo de evolución, afectación de IFP en dedo meñique y más de 2 factores diatésicos son también determinantes de un peor resultado en el tratamiento de la ED con colagenasa.

\section{CONFLICTOS DE INTERESES}

Los autores declaran no tener conflictos de intereses.

\section{AGRADECIMIENTOS}

A Isabel Millán por su inestimable ayuda en la estadística del presente trabajo. 


\section{BIBLIOGRAFIA}

I. Muñoz Peñín R, Lwoff N, Arandes Renú JM. Enfermedad de Dupuytren, una vista panorámica de nuestro entorno. Rev Iberam Cir Mano. 2011;39:126-34.

2. Azzopardi E, Boyce DE. Clostridium histolyticum collagenase in the treatment of Dupuytren's contracture. Br J Hosp Med. 20 I2;73:432-6.

3. Kaplan TD. Collagenase clostridium histolyticum injection for the treatment of Dupuytre's contracture. Drugs today. 201 1;47:653-67.

4. Loos B, Pushckin V, Horch R E. 50 years experience with Dupuytren's contracture in the Erlangen University Hospital - A retrospective analysis of 2919 operated hands from 1956 to 2006. BMC Musculoskelet Disord. 2007:8:60.

5. Crean SM, Gerber RA, Le Graverand MP, Boyd DM, Cappelleri JC. The efficacy and safety of fasciectomy and fasciotomy for Dupuytren's contracture in European patients: a structured review of published studies. J Hand Surg Br. 2011;36: 396-407.

6. Denkler K. Surgical complications associated with fasciectomy for dupuytren's disease: a 20-year review of the english literature. Eplasty. 20 1 0; $10: 1$ 1633.

7. Dias JJ, Braybrooke J.. Dupuytren's contracture: an audit of the outcomes of surgery. J Hand Surg Br. 2006;31:5|4-21.
8. de Salas Cansado M, Cuadros M, del Cerro M, Arandes JM. Budget impact analysis in Spanish patients with Dupuytren's contracture: fasciectomy vs collagenase Clostridium histolyticum. Chir Main. 20।3;32:68-73.

9. Badalamente MA, Hurst LC. Efficacy and safety of injectable mixed. collagenase subtypes in the treatment of Dupuytren's contracture.J Hand Surg Am. 2007;32:767-74.

10. Hurst LC, Badalamente MA, Hentz, VR, Hotchkiss RN, Kaplan TD, Meals RA, Smith TM, Rodzvilla J. Injectable collagenase clostridium histolyticum for Dupuytren's contracture. N Engl J Med. 2009:361:968-79.

I I. Gilpin D, Coleman S, Hall S, Houston a, Karrasch J, Jones N. Injectable collagenase clostridium histolyticum: a new nonsurgical treatment for Dupuytren's disease. J Hand Surg Am. 20 I0;35:2027-38.

12. Watt AJ, Curtin CM, Hentz VR. Collagenase injection as nonsurgical treatment of Dupuytren's disease: 8 years follow-up. J Hand Surg Am. 2010; 35:534-9.

13. Peimer CA, Blazar P, Coleman S, Kaplan TD, Smith T, Tursi JP, Cohen B, Kaufman GJ, Lindau T. Dupuytren contracture recurrence following treatment with collagenase clostridium histolyticum (CORDLESS Study): 3-Year Data. J Hand Surg Am. 2013;38: 12-22.

14. Arandes Renú JM. Aponeurotomía enzimática. Rev Iberamer Cir Mano. 20 I 4;42:3-8. 